\title{
An Approach to a Laser-Touchscreen System
}

\author{
Jeremiah Aizeboje, Taoxin Peng \\ School of Computing, Edinburgh Napier University \\ 10 Colinton Road, Edinburgh, EH10 5DT, United Kingdom \\ Jeromino2009@.gmail.com \\ t.peng@napier.ac.uk
}

\begin{abstract}
As modern day technologies advance, so have different methods in which users can interact with computers. Computers are currently being used in combination with devices like projectors for teaching and presentations, in which the mouse and the USB wireless presenter are two of the main presentation devices. However, such devices like the USB wireless presenter, is limited and cannot fully encapsulate what it aspires to simulate, a computer mouse. This device fails to simulate the movement of a mouse but may only simulate the actions of a right and left arrow key. This paper proposes a novel approach to allowing users to interact with a computer from a distance without the need of a mouse, but instead using a laser pointing device, a projector and a web camera, by developing a novel laser-touchscreen system. The test results confirmed the laser pointer could be used to simulate the movement of the mouse as well as mouse clicks with very high accuracy. It could also be potentially used in a gaming environment, especially in first person shooter games.
\end{abstract}

Keywords: Image recognition, Blob detection, Edge detection, Corner detection, Quadrilateral transformation.

\section{Introduction}

As modern day technologies advance, so have different methods in which users can interact with computers. Throughout the years, teaching and presenting devices have progressed from chalk board to white-board and now the use of projectors. Furthermore, the mouse and the USB wireless presenter are two of the main presentation devices used in combination with a projector. However, such devices like the USB wireless presenter, is limited and cannot fully encapsulate what it aspires to simulate, a computer mouse. This device fails to simulate the movement of a mouse but may only simulate the actions of a right and left arrow key. In such an environment, a camera can be used to capture the projected screen along with the laser dot. The projected screen can be seen as a "laser-touchscreen" because the laser pointer device would act as a mouse; the cursor would move to the position of the laser in relation to the projected screen. However, the USB wireless presenter, usually a laser pointer, cannot simulate the movement of a mouse but only simulate the actions of right and left arrow keys. 
The aim of this work is to improve and extend the functionalities of modern day USB wireless presenters to behave more like a computer mouse. The goal of this system would give the user more flexibility and more control over the targeted computer over any distance the laser pointer light can travel. The controller or presenter using the laser-touchscreen system would be free to move amongst the audience with confidence knowing that they don't have to rush back to the front of the audience where the computer is located, in order to do a simple mouse interaction with it i.e. clicking the play button of a video embedded within a presentation slide.

This paper proposes a novel approach to allowing users to interact with a computer from a distance without the need of a mouse, but instead using a laser pointing device, a projector and a web camera, by developing a novel screen detection method (based on a simple pattern recognition technique), a laser detection method, and an accuracy algorithm to control the accuracy of the movement of the mouse cursor. The test results confirmed the laser pointer could be used to simulate the movement of the mouse as well as mouse clicks with very high accuracy. It could also be potentially used in a gaming environment.

The rest of this paper is structured as follows. Related works are described in next section. Section 3 introduces image recognition techniques that will be used to develop the application. The main contribution of this work is presented in section 4, which introduces the novel approach, the design and implementation of the application. The testing and evaluation are discussed in section 5. Finally, this paper is concluded and future work pointed out in section 6 .

\section{Related Work}

Beauchemin [1] compared and analysed different image thresholding techniques and proposed an image based thresholding based on semivariance analysis. This method "measures the spatial variability of a variable at different scales". Semivariance thresholding proved to be highly competitive from the results gained when compared against other popular thresholding methods. Regardless of the positive results gained, the semivariance method fails when the images' background is outshined by intermittent spatial patterns.

A rectangle shape recognition algorithm was developed by Rajesh [2]. The algorithm proposes the use of a one-dimensional array to examine the rectangular shape. The algorithm requires the image to be in binary mode. Afterwards, the image would need to be rotated to a standard $\mathrm{X}-\mathrm{Y}$ axis before the rectangle testing algorithm can be run. The algorithm has been tested for three sample applications; 'Rice Sorting', 'Rectangle Shaped Biscuits Sorting' and 'Square Shaped Biscuits Sorting' as well as 'Raw Shape Sorting'. Rajesh proves the algorithm to be fast and accurate based on these applications. However, since only a one dimension array is used, only limited information can be stored. The algorithm doesn't produce accurate positions for corners, especially for unequal quadrilaterals.

Moon et al [3] proposed a method, through the use of blob detection, to help computers detect tumours in automated ultrasound images. This computer-aided detection (CADe) method was proposed to revolutionise the way hand held 
ultrasound images are carried out since the results are dependent on the user. Blob detection has made it possible for an efficiently detailed and automated ultrasound to be proposed. However, before this method can be used in a clinical environment, further work needs to be done to reduce its frames per second as well as its execution time.

There are also two existing commercial systems like electronic whiteboard and USB wireless presenter.

Electronic whiteboard (E.W.): The accuracy of this device is reliable when it has been calibrated. On the other hand it is quite costly and is not financially feasible for some commercial uses. This device works like a touchscreen; built with functionalities like mouse clicks and movement of the mouse cursor [4].

USB wireless presenter (USB W.P.): This device can be relied on when used within range of its receiver. It is built with an average range of 15 meters. It is also quite cheap and easily acquired. Its functionalities are merely pre-programmed buttons that simulates some keyboard buttons i.e. arrow keys. The USB receiver cannot work with any other pointer than the one that was built for it [5].

\section{Image Processing Techniques}

This section discusses image recognition techniques that will be used in this application.

\subsection{Image Processing}

Image processing can be defined as running a list of mathematical operations on an image in order to achieve the desired result. It has been in existence since the $1920 \mathrm{~s}$. The earliest record of a machine based image processing system was first recorded in 1952. As the development and improvement of computers grew so did this field as it became a widespread area [6].

Image processing has been used to solve several problems identified but it still has not solved some sensitive issues gathered in 1993, [7] such as:

Compression: Image compression is a technique for reducing the amount of digitized information needed to store a visual image electronically. Images are compressed to speed up transmission time from one place to another. This process causes the image to loose quality. If image processing could be used to compress a $1.2 \mathrm{Mbps}$ video stream to a desirable $1 \mathrm{kbps}$ video stream without degrading in quality then "compression" would not be a problem in image processing.

Enhancement: Image enhancement is a method used to improve the quality of an image. Attributes such as hue, contrast, brightness, sharpness etc. of an image may cause the need for an image to need enhancement. These could be seen as "degradations". The main problem of enhancement in image processing is how to remove these degradations without affecting the intended outcome of the image. Though many algorithms have been implemented but they still do not fully solve this problem. 
Recognition: Image recognition is the identification of objects within an image. This area is widely used in computer vision. Such a system should be able to recognize objects from its input parameters (analysis of the image retrieved). The difficult task would be, being able to identify different classes of objects i.e. chairs, table etc. How can one develop a general purpose system such as this? This is a question yet to be answered.

Even though all these problems and more exist, different algorithms and techniques have been developed in an attempt to address these issues. It can be argued to what extent the developed methods help in a quest for a solution.

\subsection{Blob Detection}

"Blob tracking is a method by which computers can identify and trace the movements of objects within images." [8]. A computer can find a blobs position in successive frames using this method. The idea is to track a group of pixels with similar colour or light values.

Apart from using blob detection for colour detection, Hinz [9] explains how blobs can be categorized by its geometric values:

- Blob area

- Geometric moments: centre points, and higher order moments

- Boundary length

- Parameters of a robustly fitted ellipse like:

- $\quad$ Length

- Width

- Orientation

In any case, for a specific end goal in blob tracking to be viable, blob tracking calculations need to conquer the challenges revealed by high blob interaction, for example frequent uniting and disuniting of blobs [10].

\subsection{The Canny Edge Detector}

When analysing an image, one of the popular operations carried out is edge detection. The cause of its popularity is that edges form the outline of an object, in the generic sense. An edge outlines the perimeter of an object from another object or background. Edge detection is essentially needed for accuracy in identifying various objects in images [11].

The Canny Edge Detector is a very popular and effective edge feature detector that is used as a pre-processing step in many computer vision algorithms. In 1986, John Canny characterized a set of objectives for an edge identifier and portrayed an optimal strategy for attaining them [11].

Canny also stated three problems that an edge detecting system must overcome. These are:

- Error rate — the edge detector should respond only to edges, and should find all of them; no edges should be missed. 
- Localization - the distance between the edge pixels as found by the edge detector and the actual edge should be as small as possible.

Response - the edge detector should not identify multiple edge pixels where only a single edge exists.

The Canny edge detector is a multi-step detector which performs smoothing and filtering, non-maxima suppression, followed by a connected-component analysis stage to detect 'true' edges, while suppressing 'false' non-edge filter responses [12].

\subsection{Thresholding}

"Thresholding is a non-linear operation that converts a gray-scale image into a binary image where the two levels are assigned to pixels that are below or above the specified threshold value." [13]. Thresholding is a simple method used in segmenting images. It can be used to partition out different areas of an image. This partition is dependent upon the strength of the difference between the object pixels and the background pixels [14]. Before thresholding is applied, the image is normally converted to a grey scale image. Assuming an 8-bit grey scale image conversion was used, each pixel would have a value between 0 and 255; where 0 is black and 255 is white.

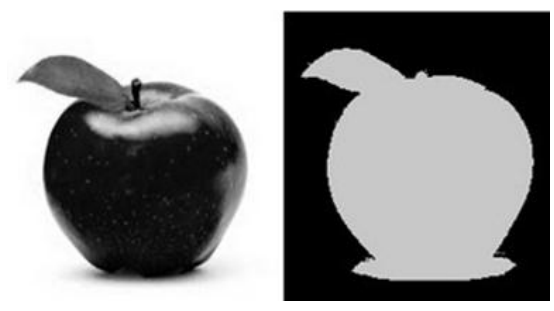

Figure 1: Thresholding applied on image. [14]

Figure 1 illustrated thresholding applied to the grey scale image on the left. The result produced on the right only contains two colours. The black colour could be classified as ' 0 ' and the non-black colour could be classified as ' 1 ' in terms of binary. When thresholding is being applied, it compares each pixel with the threshold value. If the compared pixel is less than the threshold value, that pixel is converted to 0 (black). But if the compared pixel is greater than the threshold value, that pixel would normally be converted to a non-black value (the user defines this value; between 0 255). This can also work in reverse as there are different forms of thresholding. The main goal of thresholding is to clearly separate or compress the wanted pixels from the unwanted pixels. 


\section{The Approach}

This section describes the proposed approach to allowing users to interact with a computer from a distance by using a laser pointing device, rather than a mouse.

\subsection{Introduction}

The implementation of this system is defined by its requirements. The functional requirement of this system is basically being able to use a laser pointing device to interact with a computer through the help of a web camera and a projected screen. The interaction here means the mouse must move when the laser pointing dot is moved within the projected screen and a click must be simulated when the laser pointer is turned off and on.

The accuracy of the movement is one of the main challenges. To achieve this objective, the system should answer the following two questions which are its nonfunctional requirements:

- How accurately are the four corners of the screen recognized?

- Is the position of the laser dot translated with great accuracy?

\subsection{Screen Detection}

This section proposes a method that can be used to detect the screen. When detecting the screen, the aim is to retrieve and store the coordinate of the four corners of the screen. When this coordinates have been stored, there would be no need to keep on detecting the screen. This would be costly and useless if the screen is being detected at every frame alongside with detecting the laser dot. Since the screen is inanimate and only going to be at one place, there is no need to keep on tracking so the detecting operation is carried out once. If the projector moves or is being readjusted, this method would need to be run again.

In order to detect the edges of the screen, the Canny edge detecting method was implemented as seen below.

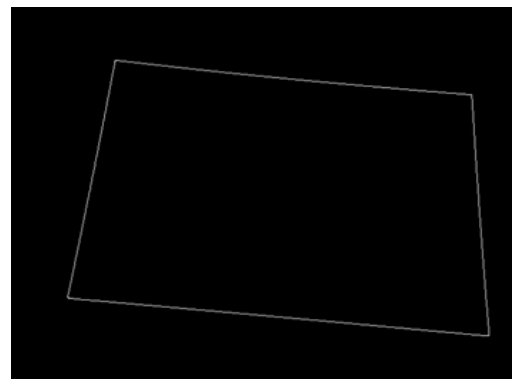

Figure 2: Canny edge applied to detect screen 
The low threshold value applied varies but is reasonably high since we are aiming to detect the most intense pixels in the image (thanks to the light from the projected screen). Figure 2 shows a black background with a white quadrilateral. This quadrilateral reveals the edges of the screen would hardly be a perfect square or rectangle.

Retrieving the four corners would require some pattern matching technique. Below are samples of patterns retrieved from a live test.

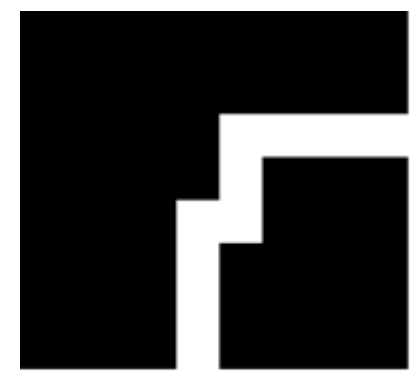

Top Left Corner

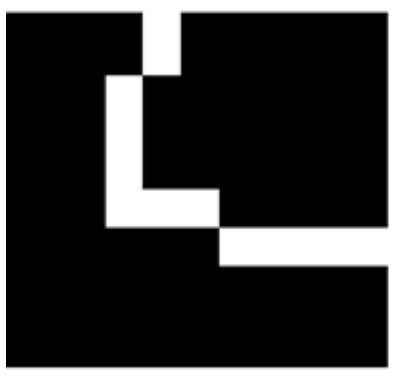

Bottom Left Corner

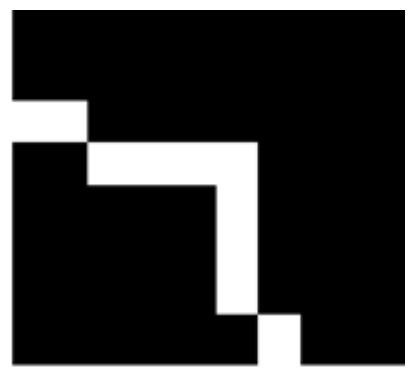

Top Right Corner

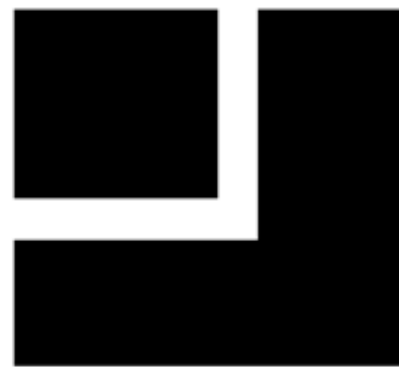

Bottom Right Corner

Figure 3: Patterns of screen corners

Then $2 \mathrm{D}$ arrays $(3 \times 3)$ to represent the corners of the binary images in figure 3 can be generated. Table 1 shows an example array for the top left corner of the image in figure 3 .

Table 1. A 2 D array $(3 \times 3)$ representing the top left corner.

\begin{tabular}{ccc}
\hline $\mathbf{0}$ & $\mathbf{0}$ & $\mathbf{0}$ \\
\hline 0 & $\mathrm{~S}$ & 1 \\
0 & 1 & 0 \\
\hline
\end{tabular}

In order to recognise the left top corner of the screen where $\mathrm{S}$ is the current pixel in question; if $S=1$ and the surrounding pixels have the values shown in table 1 then the top left corner of the screen has been found. The main goal is to check all the 8 pixels 
around a visible pixel for the pattern and if found, the $\mathrm{S}$ value is stored as a recognised corner.

\subsection{Laser Detection}

This detection method implemented the thresholding technique. Each frame received was first of all converted to a grey scale image before thresholding was applied because a grey scale image has only one channel to work with while the original colour (RGB) image would have three channels to work with. Thresholding requires one channel and the grey scale image provides just that.

Then blob detection is applied to the retrieved binary image. The tracked feature would be the intensity of the image.

The following method is adopted from the OpenCV library which is used to find a blob within a binary image:

$$
\text { cvLabel (grey, displayFrame, blobs) }
$$

In the above implementation, cvLabel takes in three parameters. The first parameter (grey) passes in a grey scale image array (IplImage). The second parameter (displayFrame) passes by reference an empty image array (IplImage) to be filled on completion of the method run. The third parameter (blobs) passes by reference an object (cvBlobs) to store the blob details found.

The centroid values ( $x$ and $y$ ) of the blob found represents the CP values used in the accuracy algorithm, proposed in next section.

\subsection{The Accuracy Algorithm}

This novel algorithm is designed specifically for this laser-touchscreen system. The accuracy algorithm can be seen as an automated screen calibration system, which is designed to answer the two questions stated in section 4.1

To help to describe the accuracy algorithm, figure 4, 5 and 6 , and relevant variables are introduced first.

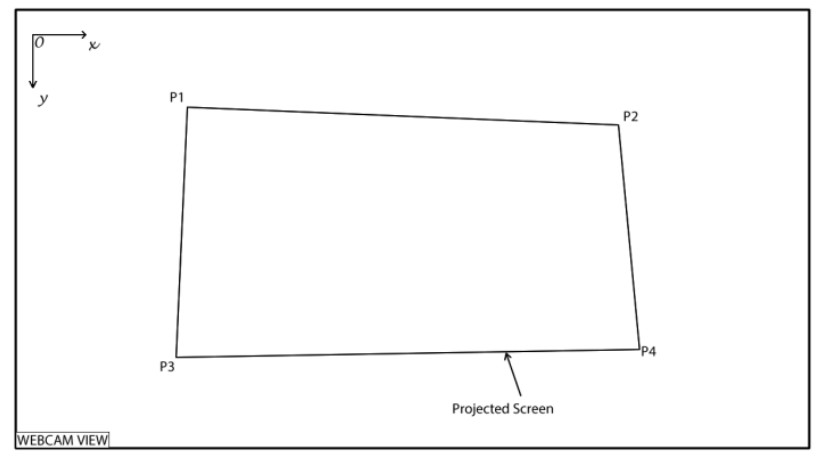

Figure 4: sketch of the projected screen on a wall 


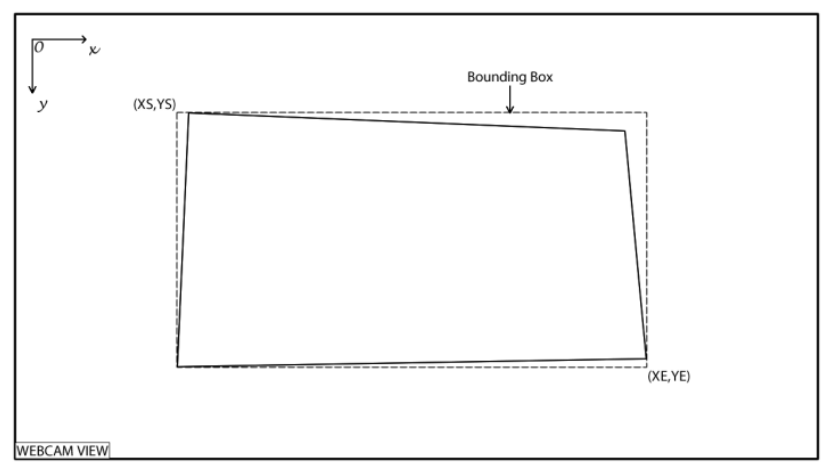

Figure 5: sketch of a bounding box around the projected screen

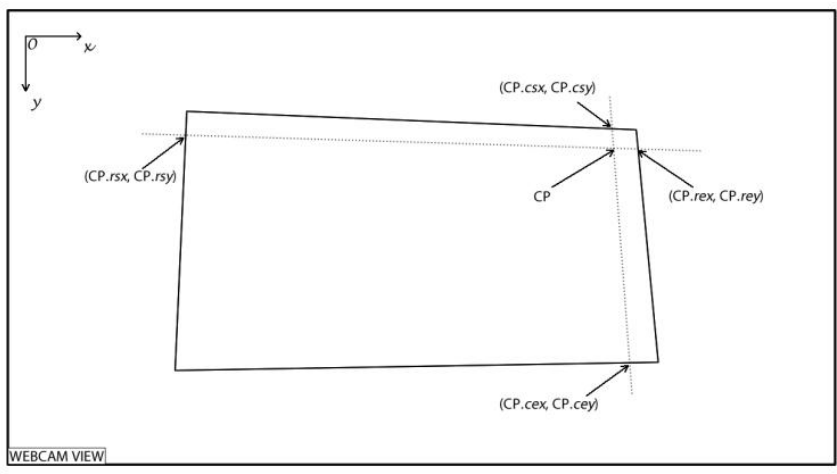

Figure 6: sketch of a laser pointer $\operatorname{dot}(\mathrm{CP})$ within the projected screen

Variables used for describing the algorithm are described in table 2, table 3 and table 4. These tables in conjunction with figure 4 , figure 5 and figure 6 help to visualise the equations that would be introduced later in this chapter. The labelled figures help to illustrate the projected screen and how certain variables were chosen.

Table 2. Variables about the project screen.

\begin{tabular}{ll}
\hline Variable & Meaning \\
\hline P1.x & top left corner X coordinate value of the screen \\
P1.y & top left corner Y coordinate value of the screen \\
P2.x & top right corner X coordinate value of the screen \\
P2.y & top right corner Y coordinate value of the screen \\
P3.x & bottom left corner X coordinate value of the screen \\
P $3 . y$ & bottom left corner Y coordinate value of the screen \\
P4.x & bottom right corner X coordinate value of the screen \\
P4.y & bottom right corner Y coordinate value of the screen \\
R $w$ & the width value of the resolution of the projected screen \\
R $h$ & the height value of the resolution of the projected screen \\
DT.x & the top difference of the X axis of P $2 . x-$ P1.x
\end{tabular}


DT.y the top difference of the Y axis of P1.y- P2.y

DL. $x$ the left difference of the $\mathrm{X}$ axis of P1.x-P3.x

DL.y the left difference of the Y axis of P3.y-P1.y

DR. $x$ the right difference of the $\mathrm{X}$ axis of $\mathrm{P} 4 . x-\mathrm{P} 2 . x$

DR.y the right difference of the $\mathrm{Y}$ axis of $\mathrm{P} 4 . y-\mathrm{P} 2 . y$

DB. $x$ the bottom difference of the $\mathrm{X}$ axis of P4.x-P3.x

DB.y the bottom difference of the $\mathrm{Y}$ axis of $\mathrm{P} 4 . y-\mathrm{P} 3 . y$

Table 3. Variables about a bounding box around the projected screen.

\begin{tabular}{ll}
\hline Variable & Meaning \\
\hline $\mathrm{XS}$ & the minimum value on the $\mathrm{X}$ axis between the $\mathrm{P} 1 . x$ and $\mathrm{P} 3 . x$ \\
$\mathrm{XE}$ & the maximum value on the $\mathrm{X}$ axis between the $\mathrm{P} 2 . x$ and $\mathrm{P} 4 . x$ \\
$\mathrm{YS}$ & the minimum value on the $\mathrm{Y}$ axis between the $\mathrm{P} 1 . y$ and $\mathrm{P} 2 . y$ \\
$\mathrm{YE}$ & the maximum value on the $\mathrm{Y}$ axis between the $\mathrm{P} 3 . y$ and $\mathrm{P} 4 . y$ \\
$\mathrm{Y} r$ & the correct value of a pixel on the $\mathrm{Y}$ axis i.e. where (P1.x, $\mathrm{P} 1 . y)$ \\
$\mathrm{X} r$ & $\begin{array}{l}\text { is seen as }(1,1) \\
\text { the correct value of a pixel on the } \mathrm{X} \text { axis i.e. where (P1.x, } \mathrm{P} 1 . y)\end{array}$ \\
$\mathrm{Y} p$ & $\begin{array}{l}\text { is seen as }(1,1) \\
\text { the bounding box value of a pixel on the } \mathrm{Y} \text { axis i.e. where }(\mathrm{XS},\end{array}$ \\
$\mathrm{X} p$ & $\begin{array}{l}\mathrm{YS}) \text { is seen as }(1,1) \\
\text { the bounding box value of a pixel on the } \mathrm{X} \text { axis i.e. where }(\mathrm{XS},\end{array}$ \\
$\mathrm{CP}$ & $\begin{array}{l}\mathrm{YS}) \text { is seen as }(1,1) \\
\text { a pixel }(\mathrm{X} p, \mathrm{Y} p) \text { within the bounding box i.e. between }(\mathrm{XS}, \mathrm{YS})\end{array}$ \\
& and (XE, YE)
\end{tabular}

Table 4. Variables about a laser pointer dot (CP) within the projected screen

\begin{tabular}{ll}
\hline Variable & Meaning \\
\hline CP.rsx & the left value of $\mathrm{X} r$ on single row of pixels \\
CP.rsy & the left value of $\mathrm{Y} p$ on single row of pixels \\
CP.rex & the right value of $\mathrm{X} r$ on single row of pixels \\
CP.rey & the right value of $\mathrm{Y} p$ on single row of pixels \\
CP.csx & the top value of $\mathrm{X} p$ on single column of pixels \\
CP.csy & the top value of $\mathrm{Y} r$ on single column of pixels \\
CP.cex & the bottom value of $\mathrm{X} p$ on single column of pixels \\
$\mathrm{CP} . c e y$ & the bottom value of $\mathrm{Yr}$ on single column of pixels \\
$\mathrm{O} t$ & the top offset for $\mathrm{CP}$ \\
$\mathrm{O} l$ & the left offset for $\mathrm{CP}$ \\
$\mathrm{O} b$ & the bottom offset for CP \\
$\mathrm{T} x$ & the translated $\mathrm{X}$-axis value of CP \\
$\mathrm{T} y$ & the translated $\mathrm{Y}$-axis value of CP \\
\hline
\end{tabular}

The algorithm translates the location of the laser dot on the projected screen to the expected mouse position on the computer.

A simple application would represent the screen as the bounding box. This would mean the only accurate point given would be the centre (XE/2, YE/2) of the screen. If 
the perimeter of the projected screen (P1, P2, P3, P4) were to be the same as that of the bounding box then that technique would work, but it may be quite difficult to achieve this depending on where the camera is placed and how the projected screen is set up.

For a single pixel row and column on the projected screen, its starting $\mathrm{X} r$ and $\mathrm{Y} r$ value would likely differ with its ending $\mathrm{X} r$ and $\mathrm{Y} r$ value. This means that in order to accurately determine the position of any pixel in question, the starting and ending values $(\mathrm{Xr} \& \mathrm{Y} r)$ of the pixel would need to be known to calculate the translated values $(\mathrm{T} x, \mathrm{~T} y)$.

Without obtaining the values of CP.rsx, CP.rex, CP.csy and CP.cey, the Tx and Ty values of $\mathrm{CP}$ cannot be calculated accurately. In order to calculate these value, the bounding box ((XS, YS) to (XE, XY)) would be used as a point of reference. The bounding box is to be used as a point of reference because each of its corners has a 90 degrees angle and none of its edges have a gradient. This would help keep the calculations simpler; not needing to take its gradient values into account. The top and left edges would be used in this case.

The idea is to make calculations based on the idea of having DT. $y$ and DL. $x$ to have a value of zero. Which would mean (P1.x, P1.y) would be equal to (XS, YS) and (P4.x, P4.y) would be equal to (XE, YE). The calculations made would need to use the absolute values of DT. $y$ and DL. $x$ to calculate their offset values.

The calculation of $\mathrm{O} l$ :

$$
\begin{aligned}
& Z= \begin{cases}\mathrm{P} 1 \cdot y, & \text { if } \mathrm{P} 1 . y>\mathrm{P} 2 . y \\
0, & \text { if } \mathrm{P} 1 . y \leq \mathrm{P} 2 . y\end{cases} \\
& W=(\text { CP. } y-Z) / \text { DL. } y
\end{aligned}
$$

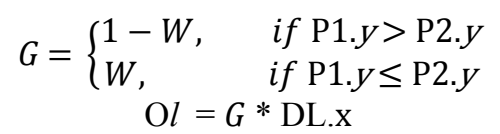

The calculation of $\mathrm{O} t$ :

$$
\begin{aligned}
& Z= \begin{cases}\mathrm{P} 1 . \mathrm{X}, & \text { if } \mathrm{P} 1 . x>\mathrm{P} 3 . x \\
0, & \text { if } \mathrm{P} 1 . x \leq \mathrm{P} 3 . x\end{cases} \\
& W=(\mathrm{CP} . x-Z) / \mathrm{DT} . x \\
& G= \begin{cases}1-W, & \text { if } \mathrm{P} 1 . x>\mathrm{P} 3 . x \\
W, & \text { if } \mathrm{P} 1 . x \leq \mathrm{P} 3 . x\end{cases} \\
& \mathrm{O} l=G * \mathrm{DT} \cdot y
\end{aligned}
$$


The calculation of $\mathrm{O} r$ :

$$
\begin{aligned}
& Z= \begin{cases}\mathrm{P} 2 . y, & \text { if } \mathrm{P} 2 . y>\mathrm{P} 1 . y \\
0, & \text { if } \mathrm{P} 2 . y \leq \mathrm{P} 1 . y\end{cases} \\
& W=(\text { CP. } y-Z) / \text { DR. } y \\
& G= \begin{cases}1-W, & \text { if } \mathrm{P} 2 . y>\mathrm{P} 1 . y \\
W, & \text { if P2.y } 1 . y\end{cases} \\
& \mathrm{O} r=G * \text { DR.X }
\end{aligned}
$$

The calculation of $\mathrm{O} b$ :

$$
\begin{aligned}
& Z= \begin{cases}\mathrm{P} 1 . \mathrm{x}, & \text { if } \mathrm{P} 3 . x>\mathrm{P} 1 . x \\
0, & \text { if } \mathrm{P} 3 . x \leq \mathrm{P} 1 . x\end{cases} \\
& W=(\text { CP. } x-Z) / D B \cdot x \\
& G= \begin{cases}1-W, & \text { if P3.x }>\text { P } 1 . x \\
W, & \text { if P3.x } \leq \text { P1.x }\end{cases} \\
& \mathrm{O} b=G * \text { DB.y }
\end{aligned}
$$

The four sets of equations above calculate the offsets values for each edge. The offset value is the space between the edge of the bounding box and the edge of the projected screen. Including the offset in the accuracy algorithm would go a long way in improving the systems accuracy.

Now that all offsets have been calculated:

$$
\begin{aligned}
& \text { CP. } r s x=\mathrm{XS}+\mathrm{O} l \\
& \text { CP.rex }=\mathrm{XE}-\mathrm{O} r \\
& \text { CP.csy }=\mathrm{YS}+\mathrm{O} t \\
& \text { CP.cey }=\mathrm{YE}-\mathrm{O} b
\end{aligned}
$$

Since each CP may have a different column height (CP.cey - CP.csy) and/or row width (CP.rex - CP.rsx) from other pixels, the above equations would be used to calculate its height and width with an end goal of solving its $\mathrm{T} x$ and $\mathrm{T} y$ values.

Once all values related to $\mathrm{CP}$ have been found, the translated position of a single pixel can now be calculated. The calculation of the translated $\mathrm{X}$-axis value of a pixel (Q) on the projected screen:

$$
\mathrm{T} x=(\text { CP.rss } /(\text { CP.rex }- \text { CP.rs } x)) * \text { CP. } x
$$


The calculation of the translated $\mathrm{Y}$-axis value of $\mathrm{CP}$ on the projected screen:

$$
\mathrm{T} y=(\text { CP.csy } /(\text { CP.cey }- \text { CP.csy })) * \text { CP. } y
$$

Translating the values of CP to (Tx, Ty) is basically what this algorithm does. The mouse cursor would be sent to the translated values of $\mathrm{CP}(\mathrm{T} x, \mathrm{~T} y)$

In conclusion, the accuracy formula wouldn't have been this accurate without adding the offsets to the edges and realising that each column of a single can have a various height values and each row of a single pixel can have a various width values. This algorithm can potentially be tweaked to triangulate a signal location when the signal is emitted anywhere within 4 receivers.

The pseudocode of the accuracy algorithm is outlined as below:

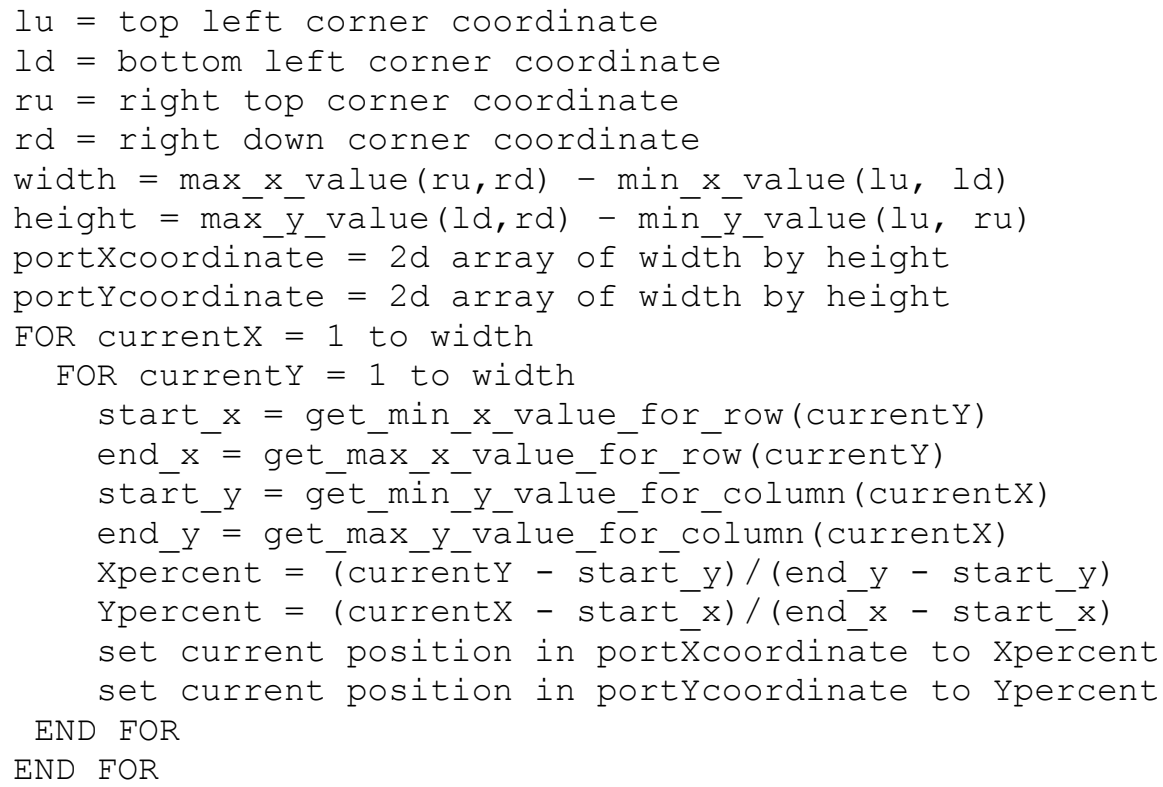

The pseudocode simulates, pre-calculates and stores every possible translated value of the laser dot (CP) in 2 different 2D arrays. This psuedocode assumes that the position of the projected screen would remain the same during the runtime of the application. Hence, there would be no need for always recalculating the same pixel multiple times if it would always return the same value. 


\subsection{Evaluation of the Accuracy Algorithm}

In order to test how effective the accuracy algorithm is, an evaluating method is proposed in this section.

Figure 7 represents a projected screen with a green laser dot and a mouse cursor. It illustrates an inaccurate system for a better explanation on how the accuracy of this system is going to be evaluated.

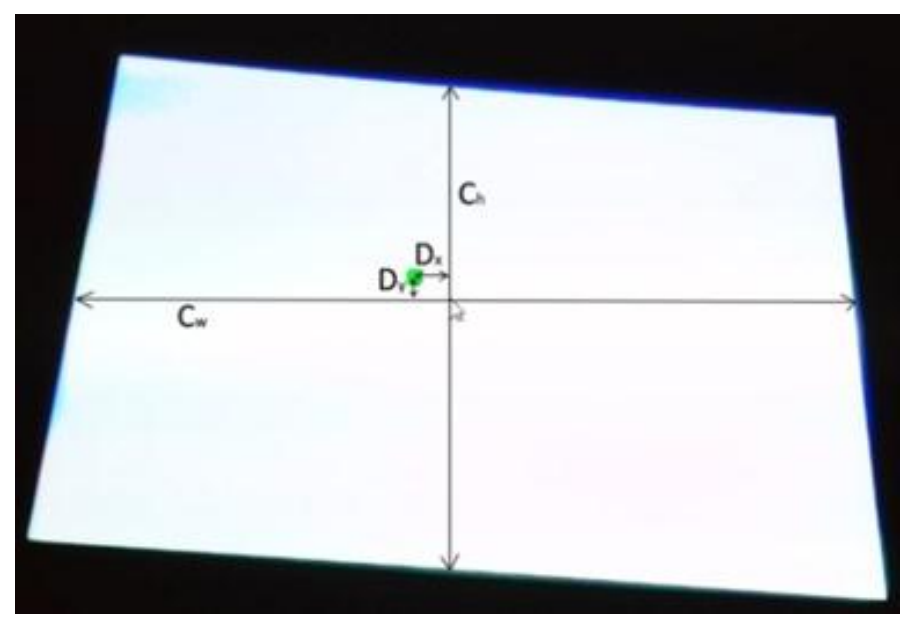

Figure 7: A projected screen; Explanation of formula used to test applications accuracy.

To evaluate the accuracy of results, the following is designed:

$$
\mathrm{S}_{\text {accuracy }}=\left(1-\left(\left(\mathrm{D}_{\mathrm{x}}+\mathrm{D}_{\mathrm{y}}\right) /\left(\mathrm{C}_{\mathrm{w}}+\mathrm{C}_{\mathrm{h}}\right)\right)\right) * 100
$$

The formula calculates how close the position of the $x$ and $y$ coordinates of the laser dot is to the position of the $x$ and $y$ coordinates of the mouse on the screen.

\subsection{Activity Design}

The diagram shown in figure 8 summarises the flow of the program. The 'search for object' process searches for either the projected screen (when setting up the program) or the laser dot (when main aspect of the program is running). When an object has been found the program analyses the object. If the laser pointer dot is detected the 'analyse object' process checks for its position (coordinates) from the camera frame and then adjusts the system by moving the mouse to its designated position. If the user sends an interrupt command (press of the Esc key) the program terminates. 


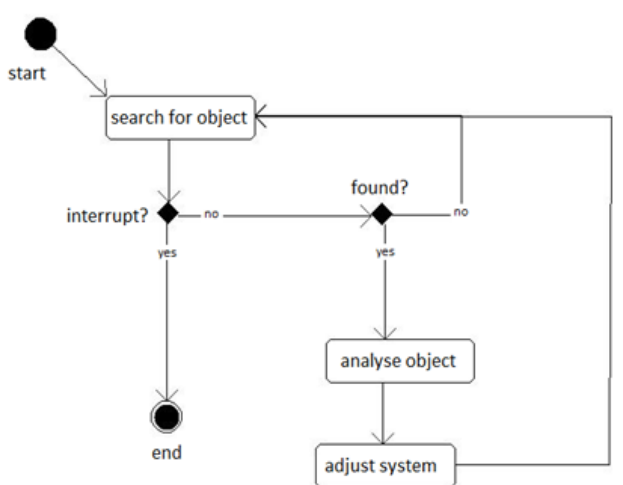

Figure 8: Flow chart for detecting an object (projected screen and laser light dot).

\subsection{Event Design}

This section elaborates on the 'adjust system' process from figure 8 . Table 5 explains the possible states and actions carried out by the program during this process.

States, variables and functions that are involved in the 'adjust system' process are defined as follows:

\section{States:}

- $\quad$ INIT - when the program is run for the first time.

- SEEN - when the laser pointer dot is seen by the camera

- NOT_SEEN - when the laser pointer dot is not seen by the camera

\section{Variables:}

- $\quad$ seen - set to true if the laser dot has been seen or else false;

- $\mathrm{dc}-$ set to true if the 'determine_click_timer()' function is called and running.

- $\quad n s c-$ this is a counter. Counts how many times there was a SEEN to NOT_SEEN state when $d c$ is true.

- range - set to true if laser dot is within the projected screen range or else false

- last_x - stores the last $\mathrm{x}$-coordinate value seen.

- last_y - stores the last $y$-coordinate value seen.

- click_interval - number of seconds to wait after a single click to determine if the user has finished clicking. (500milliseconds or 1second; user defined)

\section{Functions:}

- move_mouse $(\mathrm{x}, \mathrm{y})$ - This moves the mouse cursor to coordinate $(x, y)$ on the screen.

- determine_click_timer() - This starts a timer.

- mouse_click(type) - This simulates a mouse click. When type is:

- 1 : single left click

- 2: double left click

- 3: single right click 
Table 5. All possible laser triggered events at runtime.

\begin{tabular}{|c|c|c|c|}
\hline ID & From & Condition & Action \\
\hline \multirow[t]{2}{*}{0} & INIT & (!seen) & $($ dc $=$ false $)$ \\
\hline & Stays in the in & ate until the first appearance of the la & r pointer dot. \\
\hline \multirow[t]{2}{*}{1} & INIT & SEEN $\quad$ (seen, range $)$ & (move_mouse(xNew, yNew)) \\
\hline & $\begin{array}{l}\text { Set state to see } \\
\text { Move the mou }\end{array}$ & $\begin{array}{l}\text { fter the laser pointer has spotting the } \\
\text { o the point where the laser pointer do }\end{array}$ & $\begin{array}{l}\text { er pointer dot for the first time. } \\
\text { is currently seen. }\end{array}$ \\
\hline \multirow[t]{2}{*}{2} & SEEN & SEEN (seen, range) & (move_mouse(xNew, yNew)) \\
\hline & $\begin{array}{l}\text { Remain in see } \\
\text { currently seen }\end{array}$ & ate and move the cursor to the point & ere the laser pointer dot is \\
\hline \multirow[t]{2}{*}{3} & SEEN & (seen, !range ) & - \\
\hline & $\begin{array}{l}\text { This will occu } \\
\text { been spotted o }\end{array}$ & $\begin{array}{l}\text { ien the laser dot has been moved off } \\
\text { de the projected screen area. }\end{array}$ & projected screen area or has \\
\hline \multirow[t]{2}{*}{4} & SEEN & NOT_SEEN (!seen, range, !dc) & $\begin{array}{l}\text { (determine_click_timer(), dc } \\
=\text { true, } n s c=0, \text { last_x }=x, \\
\text { last_y=y) }\end{array}$ \\
\hline & $\begin{array}{l}\text { When the lase } \\
\text { not_seen and s } \\
\text { set } d c \text { to true a } \\
\text { something so i }\end{array}$ & $\begin{array}{l}\text { inter dot is not visible and was last sp } \\
d c \text { is false, the determine_click_tim } \\
n s c \text { to } 1 \text {. The program needs to know } \\
\text { ores the } x \text { and } y \text { coordinates of the cur }\end{array}$ & $\begin{array}{l}\text { ted in range, set the state as } \\
\text { function can be called. Then } \\
\text { the user is attempting to click on } \\
\text { nt position. }\end{array}$ \\
\hline \multirow[t]{2}{*}{5} & NOT_SEEN & NOT_SEEN (!seen, dc) & Non \\
\hline & $\begin{array}{l}\text { Remain in the } \\
\text { elapsed time } \mathrm{ft} \\
\text { the } \text { click_inter } \\
\text { This prevents } \\
\text { to cancel a clic }\end{array}$ & $\begin{array}{l}\text { seen state. The timer called by event } \\
\text { the previous click (event } 4 \text { or event } \\
\text { then the timer would come to a halt, } \\
\text { clicks (situations when the user swit }\end{array}$ & $\begin{array}{l}\text { is currently running. If the } \\
\text { to now is currently greater that } \\
\text { will be set to false, } n s c \text { to zero. } \\
\text { es off the laser pointer or wants }\end{array}$ \\
\hline \multirow[t]{2}{*}{6} & NOT_SEEN & SEEN $\quad($ seen, range,$d c)$ & - \\
\hline & $\begin{array}{l}\text { Since we are } \mathrm{s} \\
\text { this event, eve } \\
\text { elapsed time f } \\
\text { the } \text { click_inter }\end{array}$ & $\begin{array}{l}\text { rying to determine if user is attempti } \\
\text { is likely to run to simulate a left dou } \\
\text { the previous click (event } 4 \text { or event } \\
\text { then event } 8 \text { will run. }\end{array}$ & $\begin{array}{l}\text { a click, we do nothing. After } \\
\text { click or a right click. If the } \\
\text { till now is currently greater that }\end{array}$ \\
\hline \multirow[t]{2}{*}{7} & SEEN & NOT_SEEN (!seen, dc) & $($ nsc++) \\
\hline & $\begin{array}{l}\text { The laser poin } \\
\text { trying to deter } \\
\text { to move mous } \\
\text { Restart the } d e t\end{array}$ & $\begin{array}{l}\text { lot is now in the not_seen state when } \\
\text { e if the user is clicking, nsc value sho } \\
\text { vent } 6 \text { will need to be run again when } \\
\text { ine_click_timer(). }\end{array}$ & $\begin{array}{l}c \text { is true. Since it is currently } \\
\mathrm{d} \text { be incremented by } 1 . \text { No need } \\
\text { he user has finished clicking. }\end{array}$ \\
\hline \multirow[t]{2}{*}{8} & SEEN & $\begin{array}{l}\text { (range, }, \text { dc, }, \text { nsc }>0, \\
\text { nsc }<4)\end{array}$ & $\begin{array}{l}\text { (mouse_click(nsc), nsc=0, } \\
\text { dc=false) }\end{array}$ \\
\hline & $\begin{array}{l}\text { The determine } \\
\text { called with the } \\
\text { Reset } n s c \text { to ze }\end{array}$ & $\begin{array}{l}k \_ \text {timer }() \text { has run its course. The mo } \\
\text { variable passed to it. This is to deter } \\
\text { terwards. }\end{array}$ & $\begin{array}{l}\text { click(type) function can be } \\
\text { ne what kind of click is called. }\end{array}$ \\
\hline
\end{tabular}




\section{Experiments and Evaluation}

The main focus of the following experiment is to evaluate if the proposed approach is feasible as an interactive system.

The tests carried out on the application were inspired by the developed functional and non-functional requirements stated in section 4.1. Due to the facts that there are no benchmark scenarios for this kind of testing, a classic class room scenario was chosen where the device is used in a lecture room and the light/brightness of the room fails to affect the visual input of the camera e.g. the screen and the laser pointer dot. Though the presented results in this chapter had a few irregular classroom setting; the camera was placed right above (on) the projector with a slight angle. The distance from the right bottom corner of the projected screen to the centre of the projector's bulb was measured as $132 \mathrm{~cm}$ while the distance from the left bottom corner of the projected screen to the centre of the projector's bulb was measured as $151 \mathrm{~cm}$. The goal of the following test is to see how well the system works in a bad setup since it already worked perfectly fine in a good setup.

\subsection{Accuracy Test}

Once the accuracy algorithm has been run, its results can be evaluated using the method proposed in section 4.5 to evaluate how accurate the accuracy algorithm is in a real world environment.

For this test, the screen resolutions that would be used are $800 \times 600,1024 \times 768$, $1280 \times 1024$, and the camera resolutions used are $640 \times 480$ and $320 \times 240$. These resolutions are commonly found or supported by most projectors. Testing results are shown in Table $6-8$ :

Table 6. Results from an $800 \times 600$ display resolution..

\begin{tabular}{ccc}
\hline $\begin{array}{c}\text { Camera } \\
\text { Resolution }\end{array}$ & $\begin{array}{c}\text { Best Accuracy } \\
\text { Achieved }\end{array}$ & $\begin{array}{c}\text { Worst Accuracy } \\
\text { Achieved }\end{array}$ \\
\hline $640 \times 480$ & $99.99 \%$ & $97.02 \%$ \\
$320 \times 240$ & $99.99 \%$ & $96.88 \%$ \\
\hline
\end{tabular}

Table 7. Results from a $1024 \times 768$ display resolution..

\begin{tabular}{ccc}
\hline $\begin{array}{c}\text { Camera } \\
\text { Resolution }\end{array}$ & $\begin{array}{c}\text { Best Accuracy } \\
\text { Achieved }\end{array}$ & $\begin{array}{c}\text { Worst Accuracy } \\
\text { Achieved }\end{array}$ \\
\hline $640 \times 480$ & $99.99 \%$ & $97.33 \%$ \\
$320 \times 240$ & $99.99 \%$ & $96.51 \%$ \\
\hline
\end{tabular}


Table 8. Results from a 1024x1024 display resolution..

\begin{tabular}{ccc}
\hline $\begin{array}{c}\text { Camera } \\
\text { Resolution }\end{array}$ & $\begin{array}{c}\text { Best Accuracy } \\
\text { Achieved }\end{array}$ & $\begin{array}{c}\text { Worst Accuracy } \\
\text { Achieved }\end{array}$ \\
\hline $640 \times 480$ & $99.99 \%$ & $97.23 \%$ \\
$320 \times 240$ & $99.99 \%$ & $96.58 \%$ \\
\hline
\end{tabular}

The $640 \times 480$ camera resolution was able to process an average of 15 frames per second while the $320 \times 240$ camera resolution was able to process an average of 28 frames per second. Doubling the camera resolution halved the frames per seconds obtained.

The best accuracy was always achieved at the four corners of the projected screen. The reason could be because the accuracy algorithm used these points to define the screen boundaries.

On average the system can be said to be over $98 \%$ accurate. The testing results confirm the proposed accuracy algorithm provides a perfect answer to the two questions in section 4.1.

\subsection{Clicking Test}

The purpose of the clicking test evaluates how well the system simulates the clicking action by using laser pointer instead of the mouse.

There are 3 click actions involved; a left click, a double left click and a right click. A left click action is done by turning off and on the laser pointer, simulating the left click of a mouse. A double left click action is done by turning off and on the laser pointer device twice within a space of 1 second. A right click action is done by turning off and on the laser pointer thrice within a space of 1 second, simulating a right click of a mouse.

The testing results show that the approach worked perfectly on PowerPoint slides.

The application was also tested on DirectX game applications but it didn't work. Further research into a solution resulted in the need of using the DirectX API to simulate a mouse which remains as future work.

\subsection{Evaluation}

Comparing the developed screen detection method (section 4.2), against Rajeshs' [2] rectangle shape recognition algorithm, the image does not need to be rotated to detect the quadrilateral screen. Rajeshs' binary segmentation algorithm wouldn't have been able to separate the screen from the background because it requires the background colour to be known. Since this application is being developed to be used in unknown environments the Canny detector (section 3.3.) proved to be superior.

The Laser-touchscreen system (L.T.S) developed in this project can be said to be accurate based on the results from the accuracy test carried out. The camera and the laser pointer used cost less than $\$ 100$ which is relatively cheap. The interaction range of this system is dependent on the range of the laser light. The laser pointer used has a 
range 3 miles [15]. This system can simulate three mouse actions and also move the mouse cursor to the position of the laser dot. Different laser pointer devices can be used - its parts are interchangeable (P.I.).

Comparing this project with two interactive commercial devices described in section 2, E.W and USB P.W. would help to prove the usefulness of this project.

Table 9. Comparing similar devices.

\begin{tabular}{cccc}
\hline & E.W & USB W.P. & L.T.S \\
\hline Reliable & Yes & Yes & Yes \\
Cost & Expensive & Cheap & Cheap \\
Range & Short & Medium & Long \\
Accurate & Yes & N/A & Yes \\
P.I & N/A & No & Yes \\
\hline
\end{tabular}

Table 9 shows that the developed prototype (the Laser-touchscreen system (L.T.S)) can easily replace the use of an electronic whiteboard and a USB wireless presenter.

The test results illustrate that using a higher camera resolution could improve the accuracy but at the same time reduce the number of frames that can be processed in a second. It all comes down to sacrificing the application's speed against its quality (accuracy).

\section{Conclusions and Future Work}

This paper has presented a novel approach to a laser-touchscreen system, by using a laser pointer as a mouse with a web camera installed. The aim is to fully encapsulate what it aspires to simulate a computer mouse. Also the economic camera used makes the implementation of this system more attractive to potential users and perhaps financially feasible. To achieve this approach, a novel screen detection method (based on a simple pattern recognition technique), a laser detection method, and an accuracy algorithm were developed, which were successfully used to create the lasertouchscreen system. Experimental comparative studies show that in several environments with different device settings of screen resolutions and camera resolutions, on average the new system can achieve over $98 \%$ accuracy. The result from the testing and evaluation proves the use of a laser pointer as a mouse to be achievable.

Although promising, much can be done to further improve the potential of this work. For future development, further investigation and research is required to determine the best course of action on how to improve the developed accuracy algorithm so it works better under terrible setups, either by improving the existing prototype or by creating a new standalone project for the sole purpose of improving its accuracy.

Another particular interest to the authors is to extend the application to gaming, by implementing the DirectX API into the system. It is believed that it would be very user friendly when a USB device, such as a laser pointer is used in playing games, 
especially in first person shooter games or games where the user is required to aim at a particular area on the screen to achieve a goal.

Finally, it is worth noting that, once a more improved accuracy algorithm has been developed, image processing chips could be looked into and how it could be integrated into a projector alongside with a good quality camera. It would be beneficial to both the gaming industry and users if all the external components utilised in this project can be integrated into a single device, which would ensure a proper and easy setup.

\section{References}

1. Bailey, D.G.: Design for Embedded Image Processing on FPGAs. Singapore: John Wiley \& Sons (Asia) Ltd (2011)

2. Beauchemin, M.: Image thresholding based on semivariance. International Association for Pattern Recognition 34(5), pp. 456-462 (2013)

3. DIGIFLEX, TRIXES 5in1 Green Laser Pointer Pen with 5 Patterned Projection Caps, $\mathrm{http}: / /$ www.digiflex.co.uk

4. Hinz, S.: Fast and subpixel precise blob detection and attribution. IEEE International Conference on Image Processing, Vol 3, pp. 11 - 15 (2005)

5. Huang, T.S., Aizawa, K.: Image Processing: Some challenging Problems. Proceedings of the National Academy of Sciences of the United States of America, 90(21), pp. 9766-9769 (1993)

6. Luo, Y., Duraiswami, R.: Canny edge detection on NVIDIA CUDA.IEEE Computer Society Conference on Computer Vision and Pattern Recognition Workshops. Anchorage, Alaska, USA, pp.1-8 (2008)

7. Moon, W.K., Shen, Y., Bae, M.S., Huang, C., Chen, J., Chang, R.: Computer-aided tumor detection based on multi-scale blob detection algorithm in automated breast ultrasound images, IEEE Transactions on Medical Imaging.32(7), pp. 1191 - 1200 (2013)

8. OpenCV, Basic Thresholding Operations, http://docs.opencv.org

9. Parker, J.R.: Algorithms for Image Processing and Computer Vision (2nd ed.). Indiana: Wiley Publishing, Inc (2010)

10.Rajesh, F.: Rectangle shape recognition using one-dimensional array.IEEE International Conference on Computational Intelligence and Computing Research (2010)

11.SANOXY: RF Wireless remote control USB PowerPoint PPT presenter laser pointer PenSmart, http://sanoxy.com

12.Sharma, V.: A blob representation for tracking robust to merging and fragmentation.IEEE Workshop on Applications of Computer Vision. Colorado, USA, pp. 161-168 (2012)

13.SMART: SMART Board 8000 Series, http://smarttech.com

14.WaveMetrics: Image Threshold, http://www.wavemetrics.com

15.Yao, N., Liu, Z., Qian, F. \& Sun, Z.: Target Tracking Method Based on Image Patches Exemplars. Journal of Computational Information Systems 9(21). pp. 8561-8570 (2013) 